\section{POS1456-HPR THE SERUM IRON LEVEL MIGHT BE USEFUL IN DETERMINING THE SEVERITY OF MALNUTRITION IN PATIENTS WITH RHEUMATOID ARTHRITIS}

Y. Matsumoto ${ }^{1,2}$, Y. Sugioka ${ }^{3}$, M. Tada ${ }^{4}$, T. Okano ${ }^{5}$, K. Mamoto ${ }^{5}$, K. Inui ${ }^{6}$, D. Habu' ${ }^{2}$, T. Koike ${ }^{1,3} .{ }^{1}$ Shirahama Foundation for Health and Welfare, Search Institute for Bone and Arthritis Disease, Shirahama, Japan; ${ }^{2}$ Osaka City University Graduate School of Human Life Science, Medical Nutrition, Osaka, Japan; ${ }^{3}$ Osaka City University Medical School, Center for Senile Degenerative Disorders, Osaka, Japan; ${ }^{4}$ Osaka City General Hospital, Orthopaedic Surgery, Osaka, Japan; ${ }^{5}$ Osaka City University Medical School, Orthopaedic Surgery, Osaka, Japan; ${ }^{6}$ Osaka Saiseikai Nakatsu Hospital, Orthopaedic Surgery, Osaka, Japan

Background: The Global Leadership Initiative on Malnutrition (GLIM) criteria the first international criteria for diagnosis of malnutrition, was released in 2018 [1]. Patients with rheumatoid arthritis (RA) are thought to be prone to malnutrition due to decreased food intake and increased muscle catabolism caused by chronic inflammation or pain. However, there has been no report to assess the nutritional status of RA patients in accordance with the GLIM criteria. In addition, commonly used blood nutrient indicators such as albumin might not be appropriate as nutritional indicators for RA because these values are affected by inflammation.

Objectives: This study aims to examine the rates of malnutrition in RA patients according to GLIM criteria, and the relationship between blood nutrient indicators and the severity of malnutrition.

Methods: In this study, we conducted a cross-sectional survey of 135 female RA patients in 2020. According to the GLIM criteria, patients were considered to be malnourished if patients had one of the following phenotypic: (1) low body mass index, (2) non-volitional weight loss, (3) reduced muscle mass, and one of the following etiologic: (1) reduced food intake or assimilation, (2) disease burden/ inflammatory condition. Reduced muscle mass was evaluated by measuring calf circumference, and inflammatory condition was evaluated by Disease Activity Score (DAS) 28. In accordance with the GLIM criteria, the severity of malnutrition was judged as three levels: no problem, moderate, and severe malnutrition. Albumin, transthyretin, transferrin, retinol binding protein, zinc, iron, ceruloplas$\mathrm{min}$, and total cholesterol were assessed as blood nutrition indicators. Also grip strength was assessed. We compared each nutritional indicator among the three groups according to the severity of malnutrition using age-adjusted analysis of covariance, and examined the relationship between each nutritional indicato and the severity of malnutrition using receiver operating characteristic (ROC) analysis.

Results: In RA patients, $20 \%$ were classified as severe malnutrition, and $40 \%$ were moderate or more. Serum iron levels were significantly lower in the severe malnutrition group compared to the no problem group $(p=0.001)$. In ROC analysis, serum iron, zinc, albumin, and grip strength (area under curve; AUC; 0.680 $0.696,0.636,0.790$, respectively) were significant parameters for classification of moderate and severe malnutrition. Serum iron and grip strength (AUC for respective parameters were $0.741,0.747$ ) were significant parameters for classification of severe malnutrition.

Conclusion: Evaluation based on the GLIM criteria showed that about $40 \%$ of RA patients were under moderate or severe malnutrition. It was suggested that serum iron and grip strength might be useful to predict the severity of malnutrition

\section{REFERENCES:}

[1] Cederholm T, Jensen GL, Correia MITD, Gonzalez MC, Fukushima R, Higashiguchi T, et al. GLIM criteria for the diagnosis of malnutrition - A consensus report from the global clinical nutrition community. Clinical Nutrition 2019; 38: 1-9

Acknowledgements: We thank to Tomoko Nakatsuka, and the Center for Drug \& Food Clinical Evaluation, Osaka City University Hospital, for management and collection of the study data. We also thank to study participants.

Disclosure of Interests: Yoshinari Matsumoto Grant/research support from: Yamada Research Grant, Yuko Sugioka: None declared, Masahiro Tada: None declared, Tadasi Okano Speakers bureau: AbbVie, Asahikasei, Astellas Pharma Inc, Ayumi Pharmaceutical, Bristol-Myers Squibb, Chugai Pharmaceutical, Daiich Sankyo, Eisai, Janssen, Lilly, Mitsubishi Tanabe Pharma Corporation, Novartis Pharma, Ono Pharmaceutical, Pfizer, Sanofi, Takeda Pharmaceutical, Teijin Pharma and UCB, Grant/research support from: AbbVie, Eisai, Mitsubishi Tanabe Pharma Corporation and Nipponkayaku, Kenj Mamoto: None declared, Kentaro Inui Speakers bureau: Daiichi Sankyo Co. Ltd., Mitsubishi Tanabe Pharma, Janssen Pharmaceutical K.K., Astellas Pharma Inc., Takeda Pharmaceutical Co. Ltd., Ono Pharmaceutical Co. Ltd., Abbvie GK, Pfizer Inc., Eisai Co., Ltd., Chugai Pharmaceutical Co., Ltd, Grant/research support from: anssen Pharmaceutical K.K., Astellas Pharma Inc., Sanofi K.K., Abbvie GK, Takeda Pharmaceutical Co. Ltd., QOL RD Co.
Ltd., Mitsubishi Tanabe Pharma, Ono Pharmaceutical Co. Ltd., Eisai Co. Ltd., Daiki Habu: None declared, Tatsuya Koike Speakers bureau: AbbVie, Astellas Pharma Inc, Bristol-Myers Squibb, Chugai Pharmaceutical, Eisai Janssen, Lilly, Mitsubishi Tanabe Pharma Corporation, MSD, Ono Pharmaceutical, Pfizer, Roche, Takeda Pharmaceutical, Teijin Pharma, and UCB, Grant/ research support from: AbbVie, Astellas Pharma Inc, Bristol-Myers Squibb, Chugai Pharmaceutical, Eisai, Janssen, Lilly, Mitsubishi Tanabe Pharma Corporation, MSD, Ono Pharmaceutical, Pfizer, Roche, Takeda Pharmaceutical, Teijin Pharma, and UCB

DOI: 10.1136/annrheumdis-2021-eular.947

\section{POS1457-HPR GASTROINTESTINAL SYMPTOMS AND RHEUMATIC DISEASES: TWO SIDES OF THE SAME COIN}

R. Spagnuolo ${ }^{1}$, F. S. laquinta ${ }^{2}$, S. Naty ${ }^{3}$, R. D. Grembiale ${ }^{3}$, P. Doldo ${ }^{1} .{ }^{1}$ University "Magna Graecia" of Catanzaro - AOU Mater Domini - Gastroenterology Unit, Department of Experimental and Clinical Medicine, Catanzaro, Italy; ${ }^{2}$ University "Magna Graecia" of Catanzaro, Rheumatology Research Unit, Department of Health Sciences, Catanzaro, Italy; ${ }^{3}$ University "Magna Graecia" of Catanzaro, Rheumatology Research Unit, Department of Health Sciences, Catanzaro, Italy

Background: Patients with Rheumatic Diseases (RD) often show differen symptoms besides the osteoarticular ones. Healthcare workers can evaluate the impact of specific symptoms on RD patients' quality of life in order to improve global management.

Objectives: The aim of this study was to assess the presence of gastrointestinal symptoms in RD patients through Patients Reported Outcomes.

Methods: We carried out a cross-sectional study of $101 \mathrm{RD}$ patients compared with 202 healthy volunteers. Healthcare workers invited patients and volunteers to complete questionnaires on gastrointestinal symptoms, through seven domains (belly pain, constipation, diarrhea, disrupted swallowing, gas and bloating, nausea and vomiting, gastroesophageal reflux) of the Patient-Reported Outcomes Measurement Information System (PROMIS $\odot$ ).

Results: Among 101 RD patients, $34(33.6 \%)$ had a diagnosis of Rheumatoid Arthritis, $23(22.7 \%)$ had a diagnosis of Psoriatic Arthritis, 12 (11.8\%) had axial spondyloarthritis; $18(17.8 \%)$ had Systemic Sclerosis, 8 (7.9\%) were classified as Connectivitis (i.e.Lupus, Sjögren Syndrome), and finally 6 (5.9\%) had vasculitis. Median disease duration was $7 \pm 5$ years and just under half $(45.5 \%)$ of RD patients had active disease according to specific disease activity index As shown in Table 1, no significant differences were found between patients and controls with regard to demographic and anthropometric characteristics except for sex, since males were $25.7 \%(n=26)$ in RD patients and $125(61,8 \%)$ in healthy volunteers. In our study, Patients reported significantly greater gastrointestinal symptoms than healthy controls. More specifically, as shown in Figure $1 \mathrm{~A}$, median $\mathrm{T}$ score for belly pain, constipation, diarrhea and disrupted swallowing were significantly higher in the RD patients than in healthy controls $(50.2 \pm 14$ vs $41.7 \pm 12 p<0.001 ; 51.5 \pm 10$ vs $42.2 \pm 9 p<0.001 ; 45.3 \pm 8$ vs $42 \pm 5 p<0.001 ; 51.1 \pm 10$ vs $44.6 \pm 7 p<0.001)$ respectively. Moreover, also regarding others domains (gas and bloating, nausea and vomiting and gastroesophageal reflux), patients reported a median $\mathrm{T}$ score worse than healthy controls (Figure 1B).

Table 1.

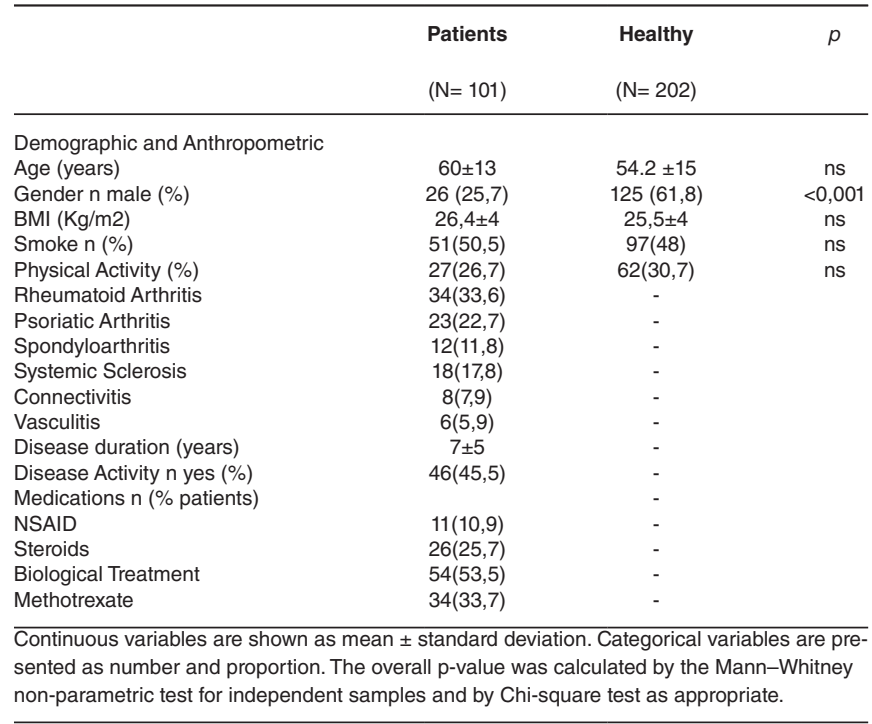



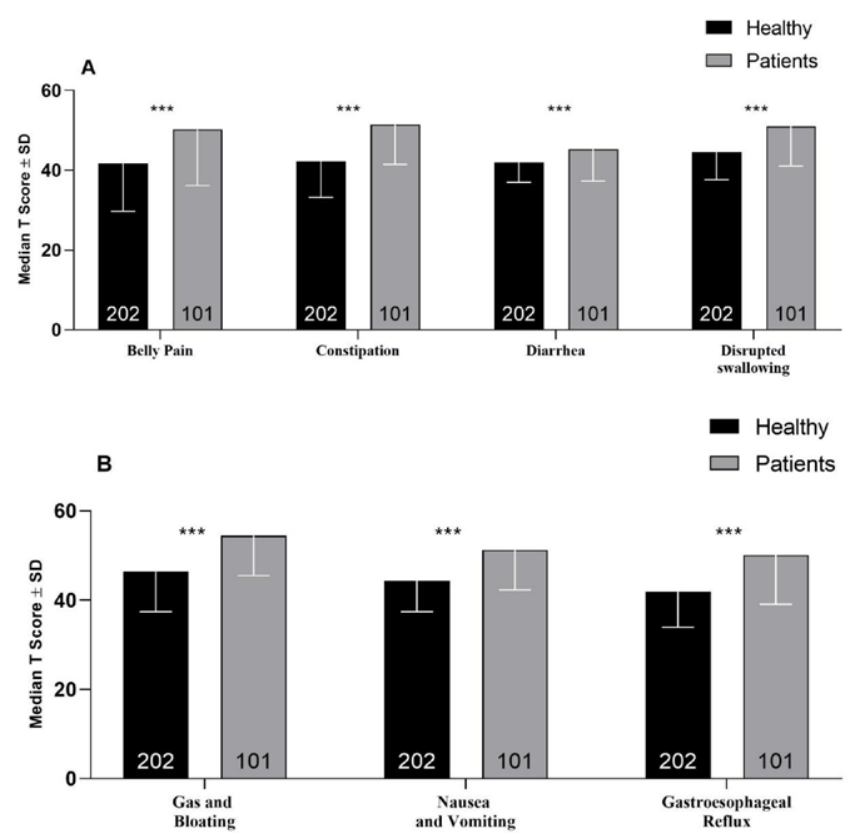

Figure 1. Median T Score stratified by study group. Data are shown as mean and standard deviation. The overall $p$-value was calculated by the Mann-Whitney non-parametric test for independent samples.

Conclusion: Firstly, this study highlights the need to investigate further the association between Rheumatic Diseases and gastrointestinal symptoms as potential patterns of underlying diseases and, secondly, the need to adopt validated questionnaires in clinical practice that can help healthcare professionals to detect underestimated symptoms.

\section{REFERENCES:}

[1] P. T. Kröner, O. A. Tolaymat, A. W. Bowman, A. Abril, e B. E. Lacy, «Gastrointestinal Manifestations of Rheumatological Diseases», Am. J. Gastroenterol., vol. 114, n. 9, pagg. 1441-1454, 2019.

[2] J. P. Witter, «The Promise of Patient-Reported Outcomes Measurement Information System-Turning Theory into Reality: A Uniform Approach to Patient-Reported Outcomes Across Rheumatic Diseases", Rheum. Dis. Clin. North Am., vol. 42, n. 2, pagg. 377-394, 2016

Disclosure of Interests: None declared

DOI: 10.1136/annrheumdis-2021-eular.1040

\section{POS1458-HPR DIGITAL RHEUMATOLOGY IN THE ERA OF COVID-19: RESULTS OF A NATIONAL PATIENT AND PHYSICIAN SURVEY}

A. Kernder ${ }^{1,2}$, H. Morf ${ }^{2,3}$, P. Klemm²,4, D. Vossen ${ }^{2,5}$, M. Meyer ${ }^{2,6}$, I. Haase ${ }^{2,7}$, J. Mucke ${ }^{1,2}$, A. Kleyer, ${ }^{2,3}$, P. Sewerin ${ }^{1,2}$, G. Bendzuck ${ }^{8}$, S. Eis ${ }^{8}$, J. Knitza ${ }^{2,3}$, M. Krusche ${ }^{2,9} \cdot{ }^{1}$ Heinrich-Heine-University Düsseldorf, Department of Rheumatology \& Hiller-Research Unit Rheumatology, Düsseldorf, Germany; ${ }^{2}$ German Society for Rheumatology (Working Group Young Rheumatology), Rheumatology, Berlin, Germany; ${ }^{3}$ Friedrich-Alexander University Erlangen-Nürnberg, Department of Internal Medicine 3 - Rheumatology and Immunology, Erlangen, Germany; ${ }^{4}$ Department of Rheumatology, Immunology, Osteology and Physical Medicine, Department of Rheumatology, Immunology, Osteology and Physical Medicine, Bad Nauheim, Germany; ${ }^{5}$ St. Elisabeth Hospital, Rheinisches Rheumazentrum Meerbusch, Rheumatology, Meerbusch, Germany; ${ }^{6}$ Universitätsklinikum HamburgEppendorf, Rheumatology, Hamburg, Germany; ${ }^{7}$ Department of Rheumatology \& Hiller-Research Unit Rheumatology, Heinrich-Heine-University Düsseldorf, Düsseldorf, Germany; ${ }^{8}$ German League against Rheumatism, German League against Rheumatism, Bonn, Germany; ${ }^{9}$ Charité Universitätsmedizin, Berlin, Department of Rheumatology and Clinical Immunology, Berlin, Germany

Background: Digital health applications (DHAs) are gaining influence and promise great potential for the monitoring and management of rheumatic and musculoskeletal diseases (RMDs).

Objectives: To analyse the impact of the COVID-19 pandemic on RMD patients' and rheumatologists' usage, preferences, and perception of digital health applications (DHAs) in Germany.
Methods: A web-based national survey was developed by the Working Group Young Rheumatology of the German Society for Rheumatology and the German League against Rheumatism. The prospective survey was distributed via social media, QR-code, and email. Descriptive statistics were calculated, and regression analyses were performed to show correlations.

Results: We analysed the responses of 299 patients and 129 rheumatologists. Most patients (74\%) and rheumatologists (76\%) believed that DHAs are useful in the management of RMDs and felt confident in their own usage thereof $(90 \%$; $86 \%$ ). $38 \%$ of patients and $71 \%$ of rheumatologists reported that their attitude had changed positively towards DHAs and that their usage had increased due to COVID-19 (29\%; 48\%).

Usage and recommendation of DHAs for both groups are shown in Figure 1:

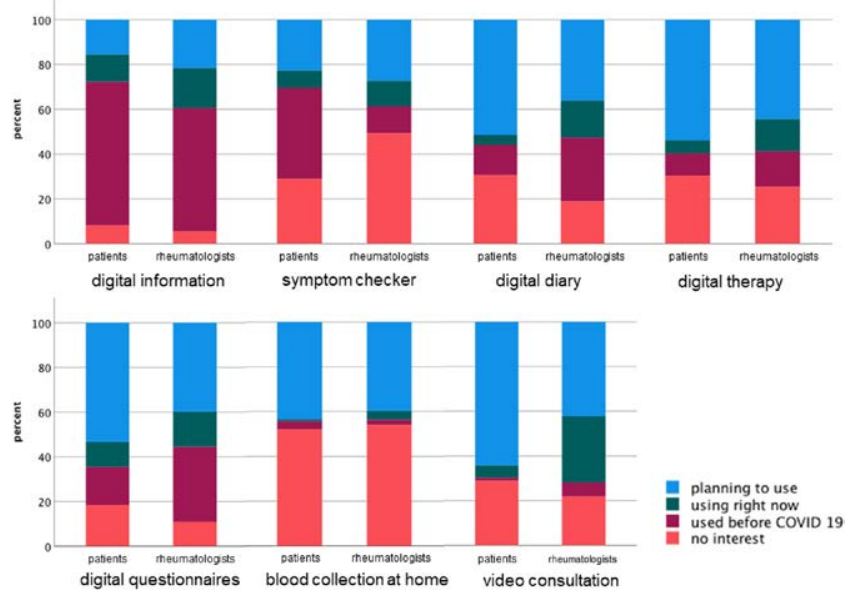

Figure 1. Usage or recommendation of digital health applications. Patients and rheumatologists were asked to indicate the specific digital health applications (DHAs) they used or were recommended.

The majority in both groups agreed on implementing virtual visits for follow-up appointments in stable disease conditions. The most reported advantages of DHAs were usage independent of time and place $(76.6 \% ; 77.5 \%)$. The main barriers were a lack of information on suitable, available DHAs $(58.5 \% ; 41.9 \%)$, poor usability (42.1\% of patients) and a lack of evidence supporting the effectiveness of DHAs (23.2\% of rheumatologists) (Table 1$)$.

Table 1. Advantages and Barriers of DHA, $n(\%)$.

\begin{tabular}{|c|c|c|c|c|c|}
\hline \multirow[t]{2}{*}{ Advantages } & \multicolumn{5}{|c|}{ Barriers } \\
\hline & Patients & Rheumatologists & & Patients & Rheumatologists \\
\hline $\begin{array}{l}\text { Location- } \\
\text { Independence }\end{array}$ & $\begin{array}{c}229 \\
(76.6)\end{array}$ & $100(77.5)$ & $\begin{array}{l}\text { Too little } \\
\text { information }\end{array}$ & $\begin{array}{c}175 \\
(58.5)\end{array}$ & $54(41.9)$ \\
\hline $\begin{array}{l}\text { Time- } \\
\text { independence }\end{array}$ & $223(74.6)$ & $94(72.9)$ & $\begin{array}{c}\text { Too little evidence } \\
\text { of benefits }\end{array}$ & $36(12.0)$ & 30 (23.3) \\
\hline $\begin{array}{l}\text { Detailed } \\
\text { documentation }\end{array}$ & $97(32.4)$ & $47(36.4)$ & $\begin{array}{l}\text { Poor quality of } \\
\text { current apps }\end{array}$ & $47(15.7)$ & $29(22.5)$ \\
\hline Cost saving & 95 (31.8) & 37 (28.7) & $\begin{array}{l}\text { Concerns } \\
\text { about data } \\
\text { protection }\end{array}$ & 52 (17.4) & $25(19.4)$ \\
\hline More information & $88(29.4)$ & $38(29.5)$ & Lack of usability & $\begin{array}{c}126 \\
(42.1)\end{array}$ & 17 (13.2) \\
\hline $\begin{array}{l}\text { Independence } \\
\text { of doctors }{ }^{+}\end{array}$ & $36(12.0)$ & - & $\begin{array}{l}\text { Lack of } \\
\text { accessibility }\end{array}$ & $4(1.3)$ & - \\
\hline More flexibility & $\begin{array}{c}107 \\
(36.8)\end{array}$ & $77(59.7)$ & High costs & $4(1.3)$ & $23(17.8)$ \\
\hline $\begin{array}{l}\text { Preparation } \\
\text { for discussion }{ }^{+}\end{array}$ & $46(15.4)$ & - & $\begin{array}{l}\text { No suitable } \\
\text { equipment }\end{array}$ & $17(5.7)$ & $11(8.5)$ \\
\hline $\begin{array}{l}\text { No advantages } \\
\text { at all }\end{array}$ & $18(6.0)$ & $1(0.8)$ & $\begin{array}{l}\text { Lack of user } \\
\text { competence } \\
\text { No Need }\end{array}$ & $\begin{array}{c}9(3.0) \\
39(13.0)\end{array}$ & $\begin{array}{c}- \\
12(9.3)\end{array}$ \\
\hline
\end{tabular}

Patients and rheumatologists were asked about the advantages and barriers of DHAs. Multiple answers were allowed. Patients had two additional potential advantages and potential barriers to choose from*. 